Artículo de Investigación | Research Article

\title{
La espiral biomimética inspiradora de retos actuales de diseño y soluciones sostenibles innovadoras | Biomimicry

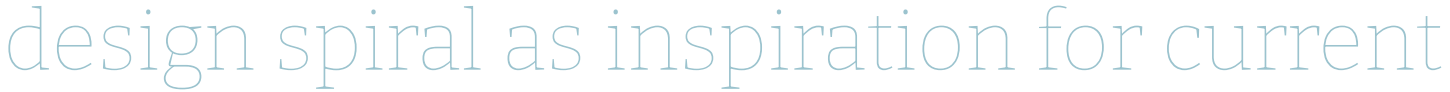 \\ design challanges and innovative

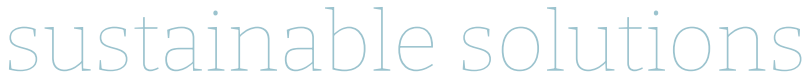

\author{
Raquel Cabrero-Olmos ${ }^{12 \star}$, Luis Calle-Sánchez ${ }^{3}$, Beatriz Rodríguez-García ${ }^{2}$ y Victoria Sevilla- \\ Lucio $^{2}$ \\ 1 Universidad de Valladolid \\ 2 Dpto. Proyectos e Investigación, Escuela de Arte y Superior de Diseño de Segovia "Casa de los Picos", España | Calle \\ Juan Bravo, 33 - 40001 Segovia. \\ hola@raquelcabrero.com; brodriguezgarcia2@educa.jcyles; mvsevillal@educa.jcyles \\ 3 Ambientólogo. Asociación Biomimicry Granada, España | Calle Real Cartuja, 7-9, local - 18012 Granada \\ biomimicrygranadaformacion@gmail.com \\ * ORCID: 0000-0002-8424-6766
}

Recibido: 20 de marzo 2021 | Aceptado: 11 de mayo 2021 | Publicado: 29 de Junio 2021 Dol: https://doi.org/10.25267/P56-IDJ.2021.11.6

\section{Resumen}

Este artículo presenta una experiencia realizada en el ámbito del diseño de producto dirigida a conocer más en profundidad qué es la biomímesis, descubrir las posibilidades que ofrece a la hora de diseñar productos más sostenibles e introducir esta inspiración en la naturaleza dentro del proceso habitual de diseño, poniendo así a trabajar de forma conjunta dos disciplinas como son la biología y el diseño.

La experiencia se ha llevado a cabo a través de varios talleres colaborativos entre futuros diseñadores de producto y asesores en biología pertenecientes a la asociación Biomimicry Granada. Se ha promovido el conocimiento y trabajo con la metodología de la espiral de diseño biomimético, a través de una aplicación guiada de la mano de expertos. En el artículo se describen detalladamente las acciones realizadas durante los tres meses de duración de la colaboración, estructuradas en fases consecutivas que se asemejan a las propuestas en la metodología (observar; definir; biologizar y descubrir; abstraer y emular; y evaluar). Se concluye con los resultados obtenidos en materia de aprendizaje e innovación sostenible, enfatizando que esta colaboración ha supuesto la apertura a nuevas disciplinas que ofrecen herramientas sostenibles e innovadoras para definir y resolver retos de diseño.

Palabras clave: Innovación, Biomimética, Pensamiento de Diseño, Proceso de Diseño, Espiral de diseño biomimético, Diseño Sostenible. 


\begin{abstract}
This paper presents an experience carried out in the field of product design aimed at knowing more in depth what biomimicry is, discovering the possibilities it offers when designing more sustainable products and introducing this inspiration in nature within the usual design thinking and process, thus putting two disciplines such as biology and design to work together.

This experience has been conducted through several collaborative workshops between future product designers and biology consultants belonging to the Biomimicry Granada association. Knowledge and work with the biomimetic design spiral methodology has been promoted through a guided application by experts. The actions carried out during the three-month collaboration are described in detail, structured in consecutive stages that resemble those proposed in the methodology (observe; define; biologize and discover; abstract and emulate; and evaluate). It concludes with the results obtained in terms of learning and sustainable innovation, emphasizing that this collaboration has opened up new disciplines that offer sustainable and innovative tools to define and solve design challenges.
\end{abstract}

Keywords: Innovation, Biomimetics, Design Thinking, Design process, Biomimetic design spiral, Sustainable Design

\section{Introducción}

"La gente que diseña nuestro mundo, por lo general, nunca ha asistido a una clase de biología, lo creas o no. Entonces son novatos en cómo funciona el mundo" (Janine Benyus).

La sociedad actual se enfrenta a grandes desafíos ecológicos, demográficos y de convivencia y la pregunta que surge es qué podemos hacer desde nuestro papel de diseñadores (Rediseñando el mañana, 2021). La cita de Janine Benyus que abre el artículo es reveladora en este sentido, poniendo en duda cómo podemos diseñar el mundo sin saber cómo funciona dicho mundo (Benyus, 1997). Define el porqué de la inquietud que nos lleva a realizar esta innovadora experiencia en el campo de la aplicación de metodologías para la creación de un futuro sostenible, ya que éste solo se generará si los creadores de nuestro mundo artificial, los diseñadores que actualmente están formándose, son capacitados para resolver problemas de diseño con principios cíclicos a imagen y semejanza de nuestra naturaleza.

Biomímesis significa literalmente imitación de la vida, combinando las raíces griegas "bio" (vida) y "mimikos" (imitación). Benyus, responsable del desarrollo del término, define biomímesis como "...una nueva ciencia que estudia los modelos naturales y los imita o toma inspiración de esos diseños y procesos para resolver problemas humanos..." (Benyus, 1997).

Las posibilidades que ofrece la biomimética al campo del diseño son incontables. Los organismosy sus ecosistemas proporcionan lecciones magistrales de diseño que pueden desembocar en soluciones innovadoras a retos de diseño a través de la analogía. La analogía biológica permite hacer una interpretación técnica de algo que ya existe en la naturaleza sin necesidad de generar una invención, establece una transferencia de conocimiento (López-Forniés y BergesMuro, 2014). En este sentido, la biología y sus especialistas son indispensables para abstraer la lógica funcional de cada uno de los organismos y sus estrategias de adaptación a sus contextos.

En base a estos referentes, se inicia un proyecto interdisciplinar, que pone en contacto directo a la asociación Biomimicry Granada, integrada por profesionales de distintas disciplinas (destacando especialmente las figuras de ambientólogo y experto en biología para la idea de nuestro trabajo), con profesores y alumnos de las enseñanzas de Diseño de Producto en 
La Escuela de Arte y Superior Diseño de Segovia, reunidos con una preocupación común, la preservación del medioambiente.

El campo de la biomimética es solo una de las posibilidades de diseño inspirado en la naturaleza; y, a su vez, abarca una amplia gama de estrategias y enfoques. varios grupos de investigación e institutos están especializados en este área, cada uno con un enfoque ligeramente diferente. (De Pauw, Kandachar, Karana, Peck y Wever, 2010). La experiencia que desarrollamos sigue las premisas del Biomimicry Institute, co-fundado por Janine Benyus en 2012. Promueve el aprendizaje del biomimetismo, estudiando y emulando las formas, los procesos y los ecosistemas naturales para crear diseños y tecnologías no contaminantes, donde no existe el concepto desperdicio solo los sistemas circulares de reutilización.

La asociación Biomimicry Granada, junto con la red de grupos locales de Biomimicry Iberia, utiliza este método para tratar de incorporar el conocimiento de la naturaleza a nuestro entorno y crear soluciones prácticas y sostenibles difundiendo la Biomímesis.

Se aprovecha la oportunidad de generar una colaboración directa con esta asociación para introducir la inspiración en la naturaleza dentro del proceso de diseño que habitualmente siguen los diseñadores de producto. La experiencia se estructura a través de una serie de talleres colaborativos entre futuros diseñadores de producto y asesores en biología pertenecientes a Biomimicry Granada. Esta colaboración interdisciplinaria no busca el dominio de muchos campos, sino la apertura a nuevas disciplinas que puedan ofrecer herramientas diferentes, más sostenibles e innovadoras, para definir y/o afrontar los retos de diseño.

Otros trabajos se han realizado ya para la introducción de la biomímesis en el campo de la formación en diseño, carente a veces de asignaturas obligatorias que cubran este aspecto de forma teórica o práctica (LópezForniés y Berges-Muro, 2014) (Santulli y Langella, 2011). Si bien el curriculum del Grado de Diseño de Producto que manejamos sí incluye temas relacionados con la biomímesis dentro de algunas asignaturas como Biónica, Ergonomía y Antropometría (de una forma más directa), o Fundamentos de Diseño (de una forma más transversal), la posibilidad de realizar una colaboración interdisciplinar con expertos en la materia es la innovación más destacada. Se promueve el conocimiento y trabajo con la metodología de la espiral de diseño biomimético, a través de una aplicación guiada de la mano de asesores en biología. A continuación se describen detalladamente las acciones realizadas durante los tres meses de duración de la colaboración, así como los resultados obtenidos en materia de aprendizaje e innovación sostenible.

\section{Intención y objetivos}

El objetivo general de la acción realizada es enraizar en los futuros diseñadores el concepto de sostenibilidad, a través de la aplicación del método biomímesis abandonando el aula y visitando el medio natural, en contacto con profesionales de la biología y otras disciplinas que colaboran con la causa a la mejora del medioambiente, en distintos espacios de colaboración, de forma conjunta e individual.

Esto se puede desplegar en diferentes objetivos específicos que enunciamos a continuación:

- Deconstruir el aprendizaje del imaginario moderno basado en la productividad y llevarlo a reintegrar al hombre a lo natural.

- Vivenciar un método creativo de resolución de problemas con principios de sostenibilidad, estableciendo analogías entre el mundo natural y el reto de diseño establecido.

- Analizar y aplicar la metodología de la espiral de diseño biomimético de forma directa de manos de un profesional que la ejerce.

- Descubrir problemas y definir retos en distintos ámbitos de la sociedad actual. 
- Crear sinergias con principios medioambientales, a través de proyectos grupales desarrollados en colaboración con diferentes disciplinas.

\section{Metodología}

La metodología utilizada en este proyecto se basa en la aplicación, a través de diferentes dinámicas participativas, de la Biomimicry Design Spiral (Fgura 1), permitiendo integrar el aprendizaje de la naturaleza en el diseño (Biomimicry Institute).

La aplicación de esta metodología en el proceso de diseño traerá consigo una nueva era de aplicaciones, tecnologías y enfoques sostenibles (Rossin, 2010). Se basa en la generación de modelos regenerativos surgidos en los sistemas naturales durante más de 3.800 millones de años de evolución de la tierra. El estudio e investigación de los mismos pueden determinar su viabilidad para la resolución de retos en el diseño o la ingeniería (Baumaster, Tocke, Dwyer, Ritter y Benyus, 2013).

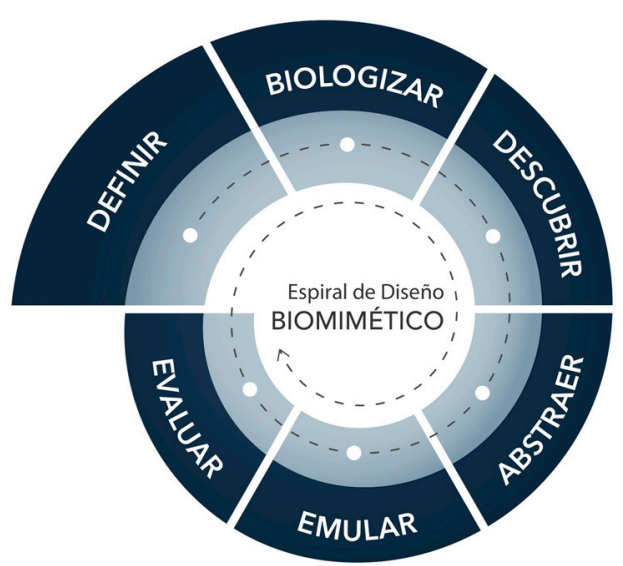

Figura 1. Proceso de diseño según la Espiral de Diseño Biomimético (Fuente: Biomimicry Institute).

La espiral de diseño biomimético se estructura en seis fases no herméticas, es decir, unas tienen relación con las otras por lo que en ocasiones, el usuario puede volver a una anterior para afrontar de mejor manera la siguiente. Este aspecto en un sentido práctico permite reevaluar conceptos tan importantes como el reto del diseño al que se enfrenta el diseñador o aportar nuevos datos biológicos que ayuden a evaluar la viabilidad de la solución creada a partir de la observación y el conocimiento de un organismo o sistema natural.

Se explican a continuación las diferentes fases de forma detallada:

10-Definir: Elobjetivo en estafase es establecer un mapa de situación de la problemática que se quiere abordar, entendiendo cuál es la necesidad que se quiere afrontar con el reto y el alcance del mismo.

2 Biologizar: En este punto se debe replantear el reto establecido en la fase "Definir" pensando cómo esas funciones identificadas se pueden encontrar en la naturaleza ya que es el lugar en el que se buscarán las posibles soluciones.

3o Descubrir: Se trata de buscar en la naturaleza las funciones que se quieren satisfacer con el diseño, es por ello que conviene dedicartiempo en los dos primeros pasos para tener un buen alcance de las funciones que permita una búsqueda fluida de alternativas en los sistemas naturales. En esta fase el conocimiento biológico prevalece a través de la observación detallada del usuario/a, la búsqueda de información bibliográfica y/o la consulta con expertos en la materia.

En este proyecto se utilizó para esta fase la herramienta Asknature (https://asknature. org/), a través de la cual se pueden encontrar soluciones en la naturaleza una vez que se han identificado una serie de funciones a solventar en la fase de "biologizar".

4 Abstraer: En esta fase el objetivo es expresar los mecanismos a través de los cuales la naturaleza observada resuelve de forma satisfactoria las funciones planteadas en el reto utilizando un lenguaje no biológico. Es importante conseguir este paso para poder emular correctamente la solución observada y aplicarla en el diseño propuesto.

5o Emular: En la emulación se deben 
aplicar todas las lecciones aprendidas en las anteriores fases en una lluvia de ideas en la cual se integren las soluciones estudiadas en la resolución de los retos a través de un diseño coherente y sostenible. En el proyecto se relacionó esta fase con los patrones unificadores de la naturaleza, una serie de directrices que la naturaleza sigue y que le permiten ser sostenible y eficiente en el tiempo como sistema. Por un lado, como criterios para entender la adecuación del diseño y por otro, para tener una mayor comprensión de cómo el diseño interacciona con el resto de elementos del sistema.

60 Evaluar: Es la fase final de la espiral, en la cual se debe evaluar la viabilidad práctica del diseño. En este proyecto por su naturaleza educativa y límite temporal de la actividad no se tuvo en cuenta esta última fase ya que no formaba parte de los objetivos descritos para el mismo.

Existen dos posibles formas de aplicar el pensamiento biomimético al proceso de diseño. La primera aproximación, "del reto a la biología", parte de un problema específico y se buscan inspiraciones biológicas para la solución. La segunda, "de la biología al diseño", se inicia con una inspiración biológica que contribuye a definir un reto de diseño (Biomimicry 3.8., 2015).
La acción desarrollada se guía por esta segunda aproximación, dedicando la parte inicial del proceso a identificar y definir un reto actual de diseño para luego pasar a la búsqueda de soluciones sostenibles innovadoras.

\section{Desarrollo}

La experiencia está contextualizada dentro de los estudios de Grado de Diseño de Producto, vinculada de manera transversal con varias asignaturas del curriculum. Integra a un total de veintidós alumnos de los dos primeros cursos y tres profesoras de sendas asignaturas. El desarrollo temporal global ha sido de tres meses de duración; la dedicación al proyecto ha sido a tiempo parcial, compaginándola con el resto de formación de cada nivel educativo en particular.

Cabe destacar un antecedente directo que da pie a lo que ahora se ha desarrollado. En 2018 se organizó un taller colaborativo con la asociación Biomimicry Granada en formato intensivo de dos días de duración (16 horas). Se comenzó con una conferencia para presentar brevemente qué es la biomímesis y sus principios fundamentales, y se dio paso al desarrollo de un taller experiencial para afianzar dichos contenidos. A través de la observación directa de elementos naturales, la realización de experimentos sencillos y el análisis de ejemplos de aplicación de la biomímesis en diferentes campos, los alumnos fueron capaces de definir proyectos basados en la naturaleza.

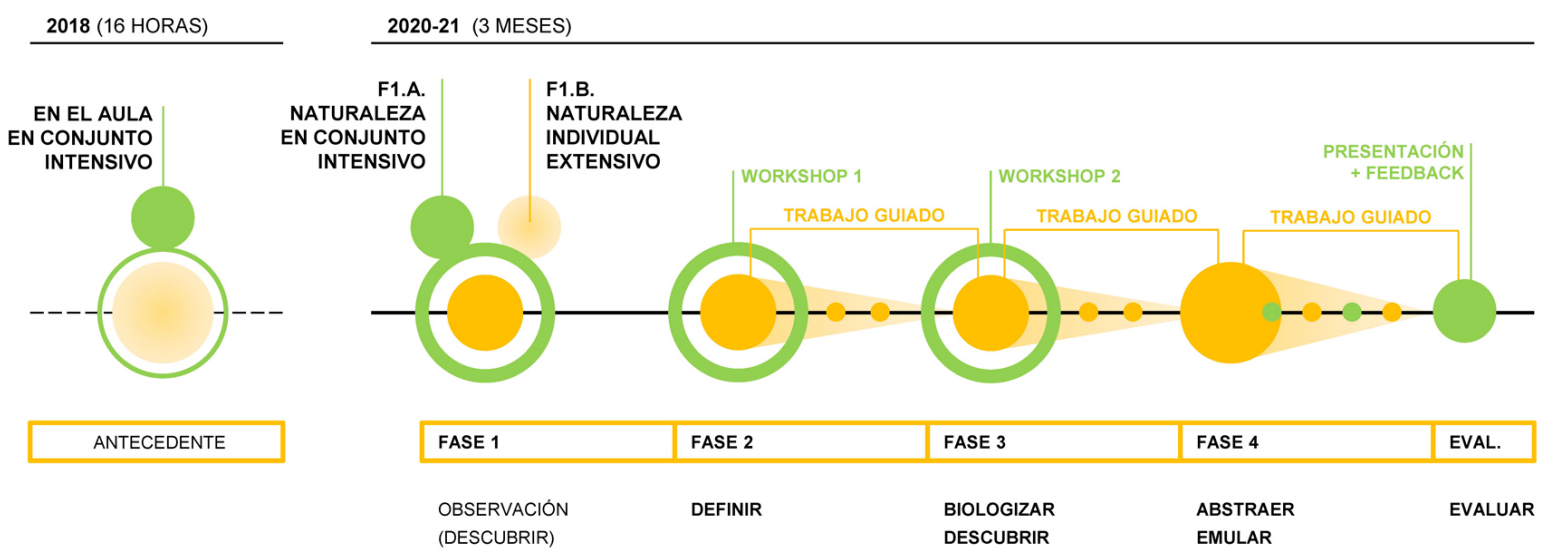

Figura 2. Cronograma de fases de la aplicación práctica. Antecedente (2018) y desarrollo de la experiencia actual (2020-2021). (Fuente: elaboración propia). 
Esta acción previa hasido el desencadenante para desarrollar ahora una experiencia colaborativa más prolongada en el tiempo que permitiese profundizar en la inspiración de la naturaleza como fuente de soluciones sostenibles, vinculando la metodología de la espiral de diseño biomimético con el proceso de diseño habitual en los estudios.

En la experiencia actual, la intervención queda organizada en cuatro grandes fases consecutivas, trabajando de forma completa un ciclo de la Espiral de Diseño Biomimético (Figura 1).

En cada una de ellas ha habido trabajo autónomo del alumno (futuro diseñador), un seguimiento continuado por parte de los profesores (diseñadores o arquitectos) y una colaboración con un experto en biología de la asociación Biomimicry Granada.

El contacto entre diseñadores y asesores en biología durante todo el proceso ha sido crucial, y se plantea como una de las mayores ventajas e innovaciones metodológicas en la vertiente del aprendizaje llevadas a cabo en esta experiencia.

Describimos a continuación las fases mencionadas, detallando las acciones realizadas en cada una de ellas, así como la implicación de los diferentes profesionales.

\section{Fase 1. Observación de la naturaleza.}

Se proponen dos actividades complementarias dirigidas a fomentar la capacidad de observación de elementos y sistemas naturales. Por un lado, se propone a cada alumno desarrollar un cuaderno de observación de la naturaleza, dibujando del natural dos días a la semana durante veinte minutos cada día. Se extiende a lo largo de 12 semanas y discurre de forma paralela al resto de acciones. Es una actividad de contacto directo con la naturaleza, que se realiza de forma individual y repetida en el tiempo.
Por otro lado, se programa una salida a la naturaleza guiada por un ambientólogo de Biomimicry Granada; se selecciona el Jardín Botánico en esta ocasión por las posibilidades que ofrece en la época del año en que tiene lugar. Esto permite realizar una observación dirigida, apoyada en dibujos, con vínculos a explicaciones concretas sobre la naturaleza por parte de un experto en la materia. Se introduce el concepto de patrones unificadores de la naturaleza (Biomimicry Institute), reflexionando de forma conjunta sobre cada uno de ellos y buscando ejemplos cercanos que los ilustren.

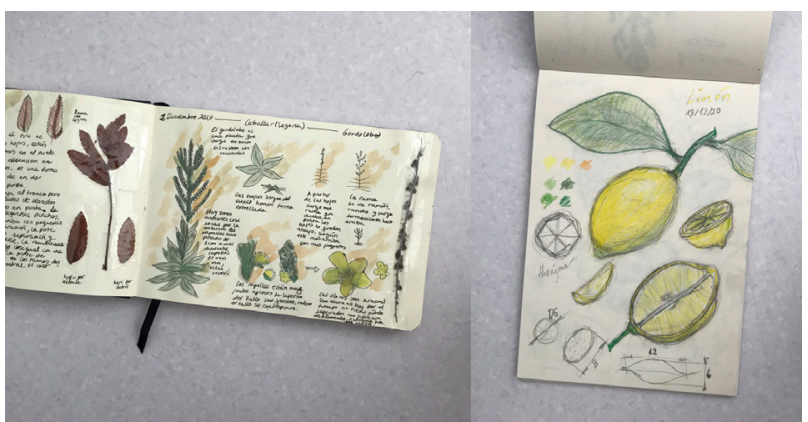

Figura 3. Extracto de dos cuadernos de observación de la naturaleza realizados por alumnas, donde destaca la capacidad de observación y análisis de los elementos naturales (Fuente: B.Virseda / T.García).

En cierto modo, esta fase de observación está relacionada con la etapa "descubrir" que propone la metodología de la espiral, y desembocará en la propuesta de retos de diseño para la continuación del proceso.

\section{Fase 2. Trabajo para la definición de un reto de diseño.}

El primer acercamiento a los retos de diseño detecta cinco grandes grupos de necesidades en la sociedad actual. Estos son: sistemas de producción y sostenibilidad; plástico y embalajes; educación e información; uso y distribución del agua; y movilidad y seguridad.

Es necesaria una mayor definición de dichos retos para poder trabajar con ellos, y es éste el objetivo de esta fase del proyecto. Se forman grupos de trabajo de dos o tres personas que se mantendrán hasta el 
final, y se concretan los retos a resolver en fases sucesivas partiendo de una plantilla que favorece el desarrollo, ordenación y concreción de las ideas a través del pensamiento visual (Figura 4a). Esta plantilla inicia la reflexión con la pregunta "¿qué quiero que mi diseño haga?", buscando más acciones a resolver que productos concretos. Deja espacio para recoger la lluvia de ideas y finaliza concretándolas en tres preguntas de diseño que comienzan por “¿Cómo podríamos...?".

Todo esto se desarrolla en un taller conjunto (nombrado como "Workshop 1" en la Figura 2), de tres horas de duración, facilitado por profesionales de Biomimicry Granada. Tras recoger los aprendizajes de la naturaleza de la fase anterior, a modo de recordatorio, este taller sirve como inyección de energía para la continuación de la experiencia.

\section{Fase 3. Biologizar y Descubrir. Inspiración en la naturaleza.}

Esta fase comienza con otro taller conjunto guiado por un asesor en biología de la Biomimicry Granada (nombrado como "Workshop 2" en la Figura 2). Otra plantilla va a servir de apoyo visual al desarrollo del pensamiento para trasladar el lenguaje de diseño a un lenguaje propio de la biología, lo que denominamos "biologizar el reto" (Figura 4b).

El formato de la plantilla es muy similar al anterior. Se fomenta la reflexión a partir de una pregunta central, "¿qué estoy buscando en la naturaleza?", que da pie a recoger verbos y acciones que traduzcan el lenguaje de diseño anterior a uno más propio de los elementos naturales. Se finaliza extrayendo tres preguntas biologizadas que comienzan con "¿Cómo la naturaleza...?".

La colaboración interdisciplinar es fundamental en esta fase para garantizar un mayor entendimiento de la parte biológica e integrar el proceso de trabajo de búsqueda de soluciones en la naturaleza.

La parte final de ese taller presenta la herramienta AskNature y enseña su funcionamiento. Se explica cómo está estructurada a partir de la taxonomía biomimética (Biomimicry Institute, 2017) y se trabaja con varios ejemplos para integrar el funcionamiento.

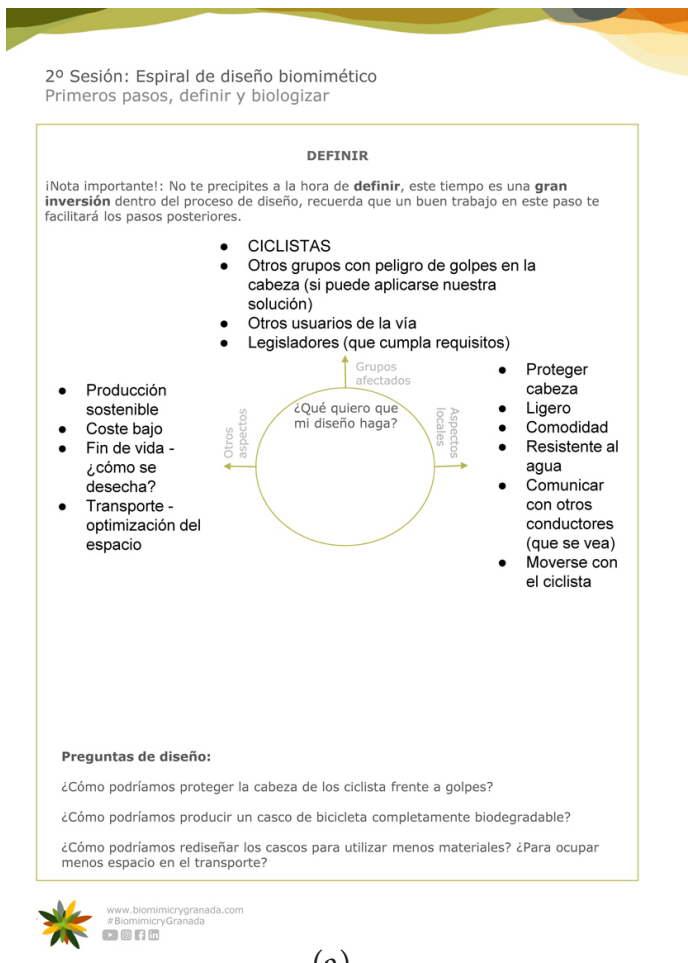

(a)

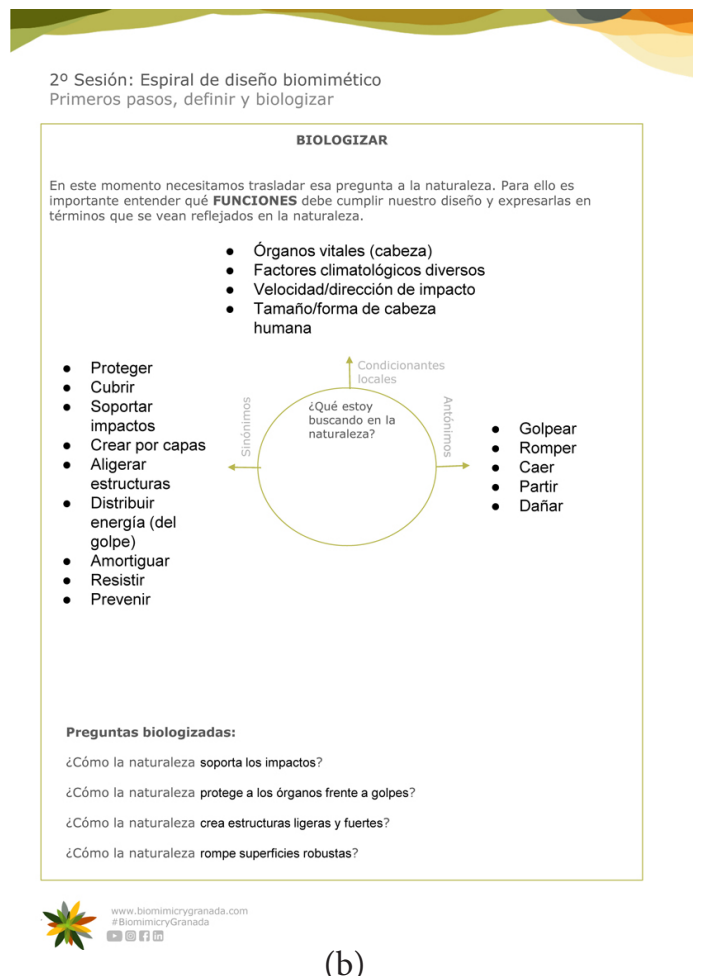

(b)

Figura 4. Plantillas de trabajo en las fases de definir (a) y biologizar (b) utilizadas durante el desarrollo de los talleres facilitados profesionales de Biomimicry Granada (Fuente: Biomimicry Granada). 
Esto permitió a los alumnos trabajar de forma autónoma en sus respectivos retos, partiendo de las tres preguntas biologizadas anteriores paradesarrollarun panelvisualque recoja los descubrimientos de la naturaleza que pueden apoyarles en el planteamiento de soluciones sostenibles. Durante esta fase, los alumnos están tutorados de forma regular por las profesoras y de forma puntual por los colaboradores externos.

Fase 4. Abstraer y Emular. Adaptación de las soluciones naturales al diseño.

Esta es la última etapa de la experiencia, dedicada a proponer una solución al reto desde el campo del diseño, inspirada en la investigación de la naturaleza de las fases anteriores.

Se desarrolla dentro del aula, con un mayor trabajo por parte del alumnado y guiada por las profesoras de las diferentes asignaturas de modo constante. El feedback de los asesores en biología es más puntual en esta fase, destinado a resolver dudas en formato diferido.

Algunos de los proyectos presentados se pueden observar en las Figuras 5, 6 y 7, asi como en la tabla resumen de resultados (Tabla 1). Cabe señalar que el desarrollo de las soluciones propuestas se ha quedado en un nivel de anteproyecto, obteniendo resultados más conceptuales que necesitarían un desarrollo técnico posterior.
Para finalizar la experiencia, se ha llevado a cabo una evaluación de las propuestas presentadas por parte de los expertos en biología y medio ambiente de la asociación Biomimicry Granada. Consideramos que esto cerraría un primer ciclo de la espiral, y serían necesarias nuevas iteraciones para seguir afinando las propuestas. Este feedback se presenta en el siguiente apartado de resultados, unido a la valoración y evaluación conjunta que hemos realizado de la experiencia.

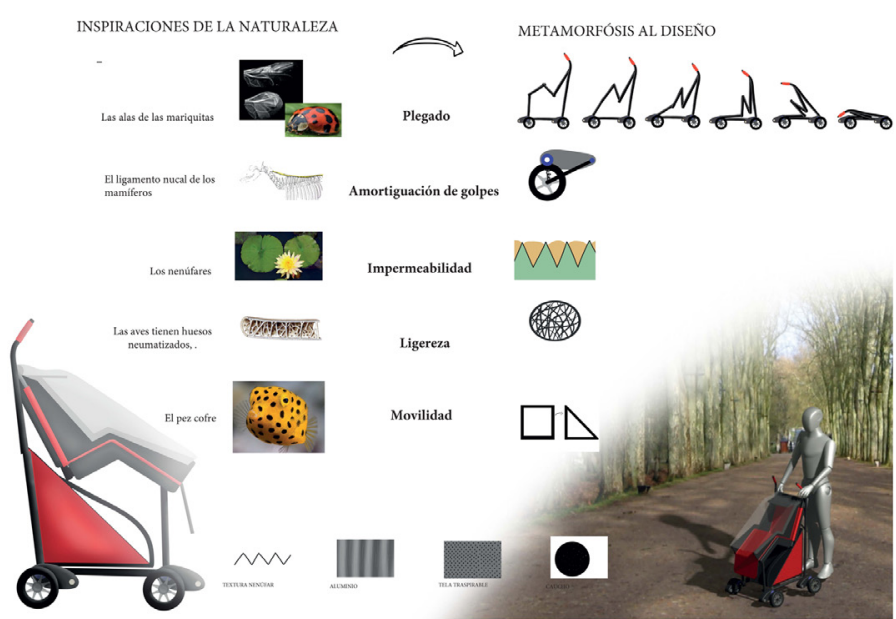

Figura 6. Proyecto "babybird". Diseño de un sistema de movilidad y transporte de niños (Fuente: D.Gómez y E.González).

\section{Resultados}

Al finalizar la experiencia, fueron presentados un total de once proyectos realizados en grupos de dos o tres alumnos; cinco se corresponden con el nivel de primer curso y seis con segundo curso de Diseño de Producto.

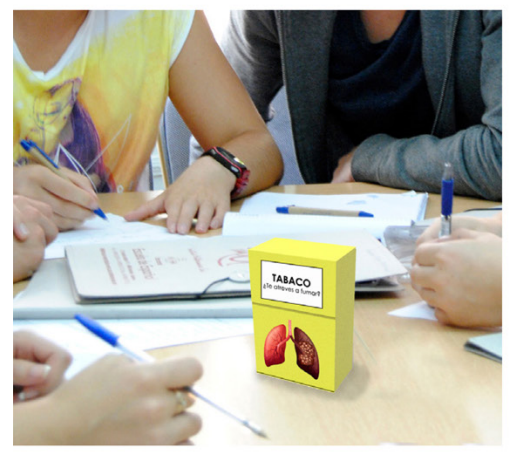

INSPIRACIÓN
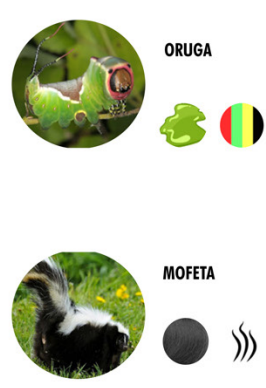

TIPOS DE CIGARROS

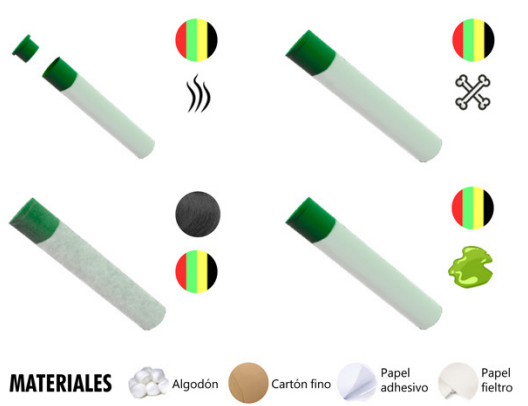

Figura 5. Proyecto "bimar". Diseño de paquete experimental de tabaco dirigido a concienciar sobre los peligros del mismo (Fuente: R.Martín y M.Centeno). 
Se puede decir que la experiencia ha sido satisfactoria en su globalidad. Todos los alumnos han concluido el proceso de resolución del reto guiados por la espiral de diseño biomimético. De los once proyectos, solo tres han trasladado las observaciones de la naturaleza de forma dudosa; aun habiendo trabajado la fase de biologizar y descubrir de forma correcta, se considera que la abstracción y emulación de dicha inspiración puede ser mejorada. Por tanto, ocho proyectos tienen propuestas validadas, que podrían continuar evolucionando en ciclos sucesivos de la espiral.

Cabe realizar algunas consideraciones a la hora de visualizar los resultados:

- Es una experiencia que se centra más en el aprendizaje de la metodología, no tanto en el producto final conseguido.

- El tiempo de desarrollo de la experiencia colaborativa ha sido breve. Como consecuencia, la resolución de los retos se ha quedado en un nivel conceptual, siendo conscientes que cada uno de los casos propuestos necesitaría más desarrollo para profundizar en la observación de la naturaleza y resolver cuestiones técnicas y tecnológicas.

- En general, debido al reto de diseño propuesto en cada caso, ha sido difícil concretarenunasolaaplicación biomimética. Esto ha derivado en un incremento de la complejidad de los proyectos.
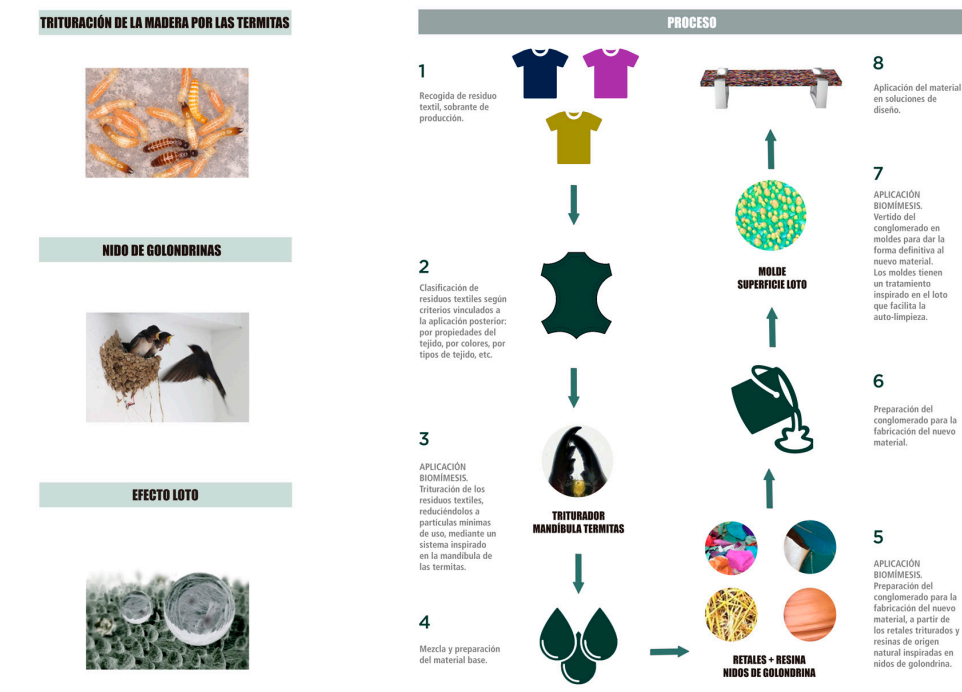

Figura 7. Proyecto "retail system". Diseño de un sistema de aprovechamiento de residuos textiles para la creación de un nuevo material (Fuente: A.Gallardo, J.Santos y B.Vírseda).
En la tabla 1 se presenta el resumen de resultados más interesantes desde el punto de vista de la biomímesis. Se indican las principales características del proyecto, la inspiración biomimética y los comentarios, a modo de feedback, que apoyarían a seguir desarrollando el proyecto hacia una solución sostenible. Se identifica cada comentario con la fase del proyecto a la que corresponde, descrita con anterioridad: Fase 1. Observación; Fase 2. Definir; Fase 3. Biologizar y Descubrir; Fase 4. Abstraer y Emular; y se utiliza la expresión 'Profundidad en el Estudio' (PE) para indicar sugerencias de mejora en una posible continuación del proyecto.

Al finalizar la experiencia interdisciplinar, se realizó una encuesta a los alumnos participantes con la intención de valorar su percepción sobre el desarrollo y aplicación de la biomímesis en el contexto del diseño de producto. De ella se destaca que el 94\% ha considerado esta colaboración 'muy útil' para el desarrollo de su profesión y todos ellos manifiestan la intención de volver a aplicar la metodología en un futuro. Un 94\% lo percibe como un incentivo para la búsqueda de soluciones sostenibles en sus futuros proyectos, considerando que ha tenido mayor incidencia en la 'definición de retos y nuevos productos' (93\%), en la 'búsqueda de la solución formal' (81\%) y en la 'investigación de soluciones técnicas' (70\%). Por último, un 53\% considera que la espiral de diseño biomimético es 'fácil de aplicar', mientras que el resto (47\%) valora el apoyo recibido a través de la formación teórica inicial, del proceso colaborativo guiado y de la revisión de ejemplos existentes.

\section{Conclusiones}

Se consideran alcanzados los objetivos que se planteaban al comienzo de la experiencia, generando en los estudiantes un interés más consciente en la sostenibilidad y produciendo un acercamiento al mundo natural como elemento inspirador de retos actuales de diseño y soluciones sostenibles innovadoras. 
Tabla 1. Resumen de los proyectos más interesantes desarrollados por los alumnos, desde el punto de vista de la biomímesis.

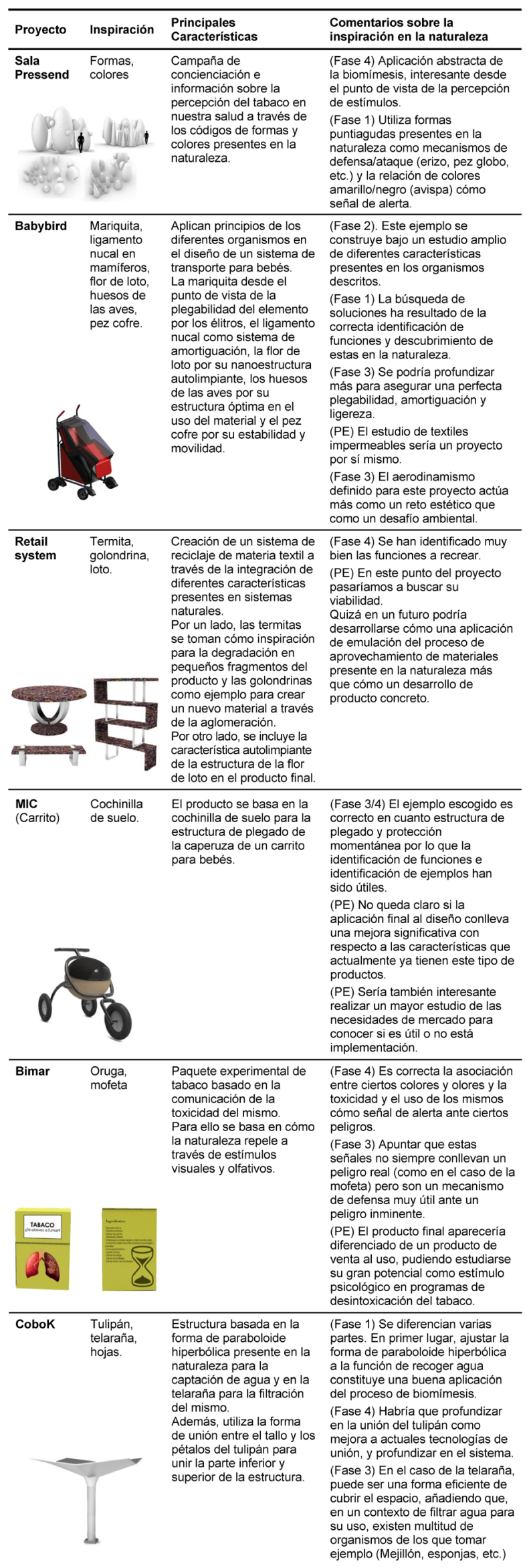

Varios aspectos podemos resaltar como conclusión de la experiencia colaborativa Llevada a cabo.

- Ha existido una buena comunicación enseñanza-aprendizaje en los distintos contextos de comunicación, especialmente remarcar lo positivo de la enseñanza en el medio natural. La salida al jardín botánico resultó útil para el desarrollo de la capacidad de observación y la comprensión de conceptos como los patrones unificadores de la naturaleza.

- En cuanto a la metodología, se ha comprobado que la aplicación de la biomímesis como proceso de diseño mejora la capacidad de observación y ayuda a los estudiantes a desarrollar la creatividad en el diseño, enriqueciendo los procesos ya conocidos

- Los resultados evidencian que para la consecución de resultados viables en la práctica, el proyecto requiere más tiempo de desarrollo en algunas de sus fases. Una de las mayores problemáticas en la consecución del proyecto fue la de definición de un reto de alcance preciso por parte de los alumnos/as.

- Destaca la colaboración interdisciplinar, que favorece el entendimiento y el encuentro exitoso de soluciones. El trabajo colaborativo generó interesantes debates grupales en el desarrollo de las propuestas para alcanzar la definición del proyecto de forma colectiva de todos los participantes implicados, en la experiencia y en cada equipo. Se han creado sinergias entre ambas disciplinas, encontrando una nueva aplicación a los conocimientos biológicos y una nueva fuente de inspiración en el entorno del diseño.

Para finalizar y a modo de reflexión final, se entiende esta experiencia como un avance en dos caminos que es preciso recorrer en este momento: Por un lado, la colaboración a la urgencia climática y la necesidad de abordar medidas de atención al cambio climático desde todas las instituciones. Por otro, la renovación de metodologías en estudios superiores, basadas en métodos de trabajo eminentemente prácticos, para definir el contacto y entendimiento del medio al que pertenecemos: la Naturaleza. 


\section{Referencias}

Innovation Inspired by Nature. (s. f.). AskNature. Recuperado 19 de marzo de 2021, de https://asknature.org/

Baumeister, D., Tocke, R., Dwyer, J., Ritter, S. y Benyus, J. (2013). Biomimicry Resource Handbook: A Seed Bank of Knowledge and Best Practices. Missoula: Biomimicry 3.8.

Benyus, J. (1997). Biomímesis. Innovaciones inspiradas por la naturaleza. Barcelona: Tusquets.

Biomimicry Institute. Recuperado 19 de marzo de 2021, de https://biomimicry.org/

Biomimicry Institute (2017). Ask Nature. The biomimicry taxonomy. Recuperado 19 de marzo de 2021 de: <https://toolbox.biomimicry.org/wpcontent/uploads/2017/03/Taxonomy_ Explainer_2017diagram.pdf>

BIOMIMICRY INSTITUTE. Biomimicry toolbox. Recuperado de: <https://toolbox.biomimicry. org/> [Consulta: 19/03/2021]

BIOMIMICRY 3.8. (2015). Perspectivas de diseño. Recuperado 19 de marzo de 2021 de: https://biomimicry.net/

De pauw, I., Kandachar, P., Karana, E., Peck, D., \& Wever, R. (2010). Nature inspired design: Strategies towards sustainability. ERSCP-EMSU Conference on Knowledge collaboration \& Learning for Sustainable Innovation, p. 1-21.

López Forniés, I., \& Berges-Muro, L. (2014). Approach to biomimetic design. Learning and application. DYNA, 81(188), 181-190. https://doi.org/10.15446/dyna.v81n188.41671 Rediseñando el mañana, Dir. Pedro Aguilera. Producciones Belino. 2021. Recuperado de <https://youtu.be/zc1U74UCEW4> [Consulta: 23/04/2021]
Rossin, K. J. (2010). Biomimicry: nature's design process versus the designer's process. Design and Nature V. Published. https://doi.org/10.2495/dn100501

Santulli, C., \& Langella, C. (2010). Introducing students to bio-inspiration and biomimetic design: a workshop experience. International Journal of Technology and Design Education, 21(4), 471-485. https://doi.org/10.1007/s10798010-9132-6 